\title{
3D/4D Volumetry in the Second and Third Trimester of Pregnancy
}

\author{
Sándor Nagy \\ Petz Aladár County Teaching Hospital of Semmelweis University, Gyõr, Hungary
}

\begin{abstract}
Purpose of the review: The technological improvements have greatly progressed on three-dimensional ultrasonography. This review summarizes these technical changes and the latest advances of their use in prenatal diagnosis.
\end{abstract}

Material and methods: Review of the literature.

Results: The new technical aspects of the volumetry, improvement of different render modes, the postprocessing modalities, and innovations on volume calculations are extensively described, as well as detailed, organ based diagnosis of different malformations in the second and third trimester are summarized.

Conclusion: Though the traditional 2D ultrasound with high resolution provides a great diagnostic tool in detection of fetal malformations, there is no doubt that the 3D/4D technique offers a new power in prenatal diagnosis. Three-dimensional ultrasound can assist in the diagnosis of different, rare malformations because it offers a potential benefit of understanding spatial relationships of normal and abnormal fetal anatomy.

Keywords: Three-dimensional ultrasound, prenatal diagnosis.

\section{INTRODUCTION}

Rapid development of computer technology of 3D ultrasound has provided progress in the different render modes. With these advances of obstetric imaging a spectrum of fetal abnormalities can be recognized prenatally. In certain fetal anomalies this "fine tuned" diagnosis provides an opportunity with adequate time to make a final decision and give genetic counseling. The $4 \mathrm{D}$ technique allows a real time visualization of fetal structures in particular observing the movement and behavior of the fetus, especially in the second and third trimester of pregnancy.

\section{TECHNICAL ASPECTS-IMPROVEMENT OF DIFFERENT RENDER MODES}

\section{Multiplanar Modes}

To obtain a good 3D picture is crucial to determine the contents of the render box and to use the appropriate render mode. Using sectional planes, 3 orthogonal planes (longitudinal, transverse and coronal) provide access and display of "any-plane" inside the acquired volume data set (volume block) by rotations and translations. After the volume has been obtained different display modes can be used, which determine how certain 3D structures are visualized: In surface, transparent or invert mode. The mixture of two selected modes provides the optimal image quality.

In grey scale rendering the surface of the fetus surrounded by fluid or a hypoechoic region is visualized. The hypoechoic region is characterized by selecting the threshold parameter, which is also an important factor to determine the quality of the picture.

\section{Surface Modes}

This mode is useful to examine the surface of the fetus. In the surface texture mode the values of the surface are identical with the gray values of the original scan, while in the smooth surface mode the structures are smoother. In the light and gradient light modes the structures oriented to the viewer are displayed brighter, and objects distant from the examiner are displayed darker, as if being illuminated from a spot light source (Figs 1A to D). These modes are useful when examining the fetal face, neck, limbs, abdominal wall, umbilical cord, and the genitals, where it is important to enhance the tissue borders.

\section{Transparent Modes}

In the maximum mode the maximum gray values of the ROI (region of interest) are displayed. This part of the application is the best for examining bony structures, especially the spine, the cranial bones and the fetal palate. In the minimum mode the minimum gray values of the ROI are displayed, so the vessels and cystic structures are shown. In the $X$-ray mode all gray values are displayed, but to get the best result and enhance the contrast, the depth of the ROI should be adjusted as low as possible. In these transparent modes images require a certain number of different views shown in a rotating cine sequence.

\section{Inversion Mode}

The inversion mode allows for the visualization of hypoechoic structures, which are difficult or impossible to display with conventional ultrasound techniques. Render algorithms analyze 

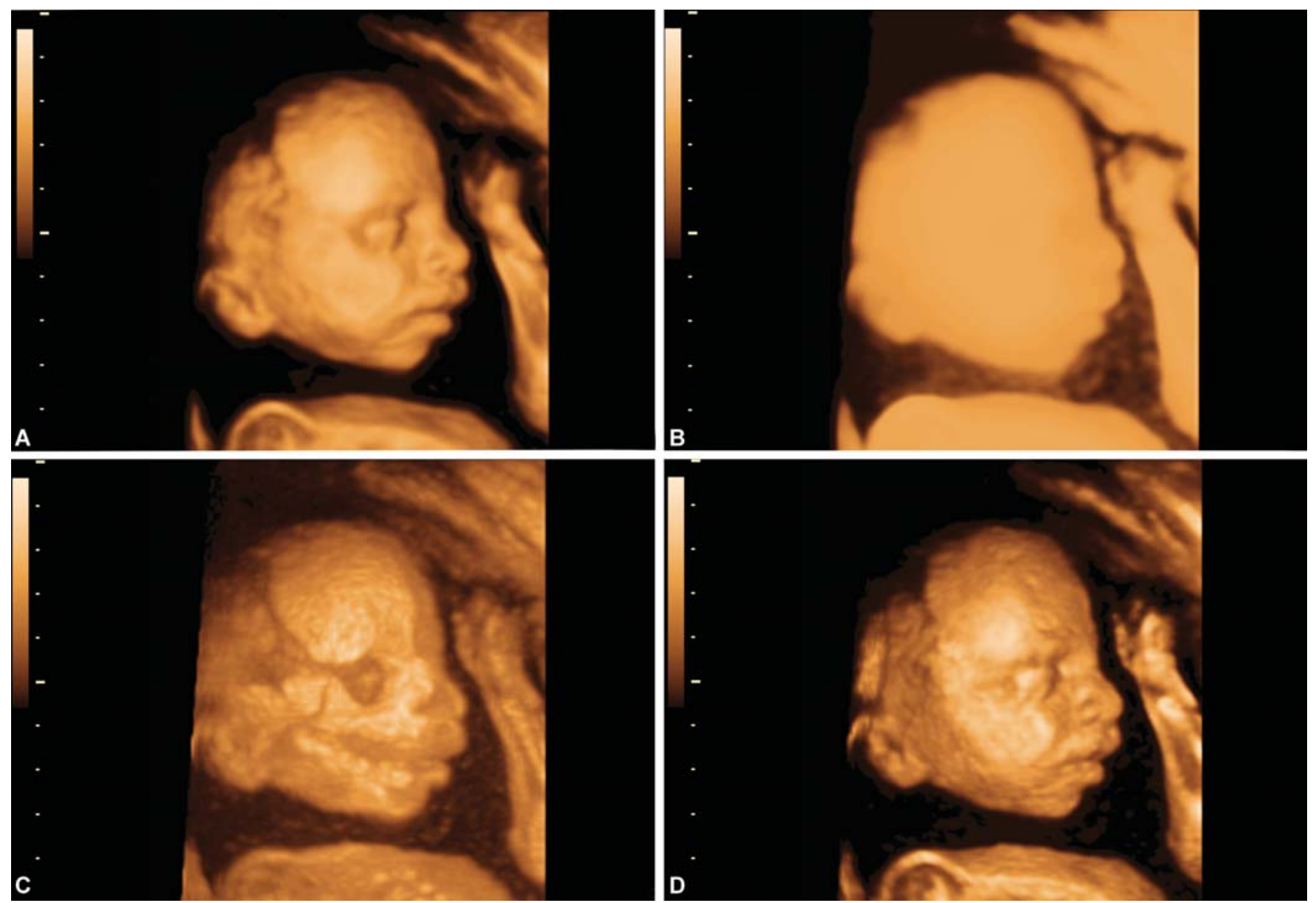

Figs $1 \mathrm{~A}$ to $\mathrm{D}$ : The same object using different rendering modes. Differences between the surface and transparent (maximum) modes is clearly visualized

the target 3D volume, displaying hypoechoic structures in a clear inverted surface rendered display, while simultaneously removing information from the surrounding tissue. Its clinical application is useful for examining the fetal stomach, ventricles of the brain, and fetal heart in combination with Doppler technique (Figs 2A and B).

\section{GLASS BODY}

By combining information received from the Power/Color Doppler and tissue there can be a perfect analysis of the vascularization of the region of interest. This color render mode feature gives the possibility to improve the ultrasound evaluation of the vascularization of any lesion.

\section{D POWER / COLOR DOPPLER}

By combining information received from the Power/Color Doppler and from different tissues one can perfectly analyze the vascularization of a region (Fig. 3).

\section{POSTPROCESSING MODALITIES}

The biggest advancement of 3D/4D ultrasound is that the volume can be manipulated in different ways depending on the objective of the diagnosis. By reslicing and rotating acquired volumes in any direction we can translate the scan into the most appropriate position. This cannot be achieved using 2D ultrasound. We can also change the brightness, contrast and color to make it easy to display any object in optimal conditions.

\section{MULTIPLANAR REFORMATING [MULTISLICE VIEW OR TOMOGRAPHIC ULTRASOUND IMAGING (TUI)]}

Multiplanar reformatting makes an analysis and documentation of dynamic studies easier by providing a simultaneous view of multiple slices of a volume data set. The simultaneous display of all three sectional planes (The Multislice View of Medison or Tomographic Ultrasound Imaging display mode of GE-General Electric) allows us to display the fetal organs as a tomographic survey. 

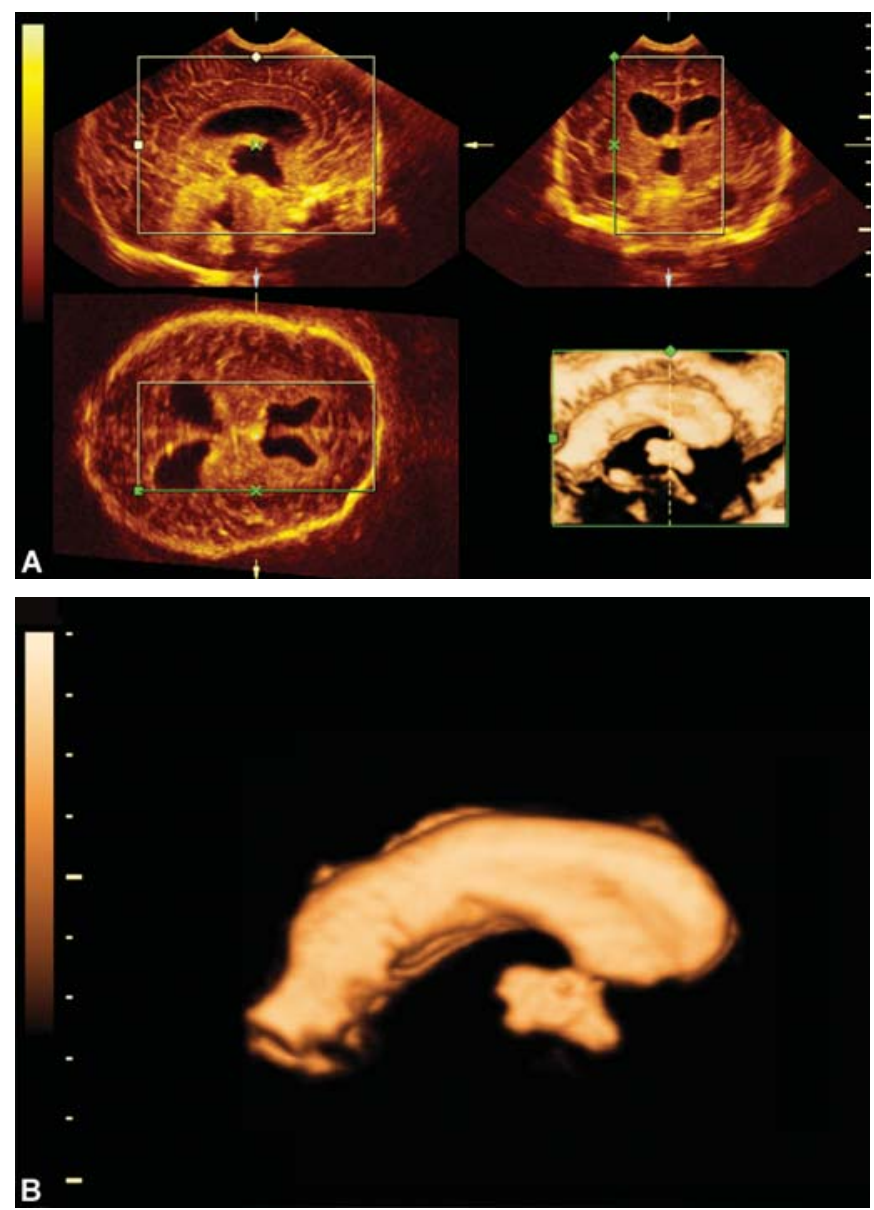

Figs 2A and B: Invers mode for assessment of fetal ventricules, and magic cut for clear the structure

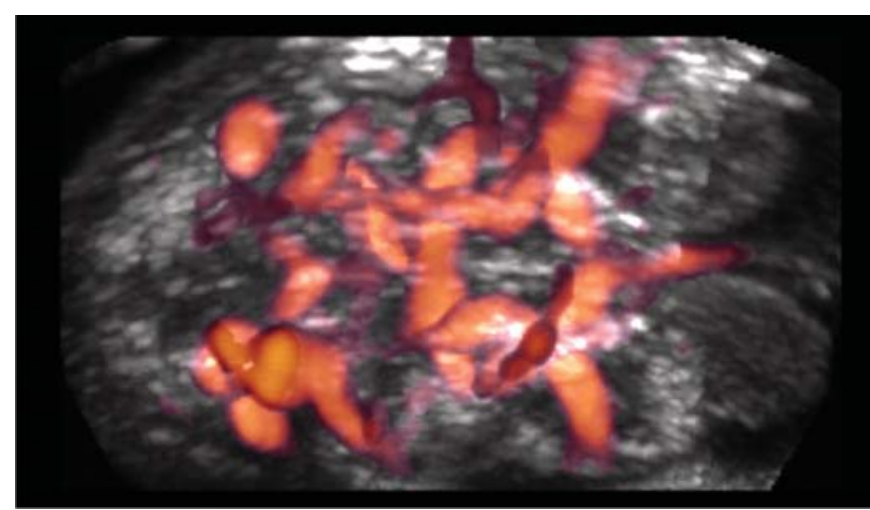

Fig. 3: 3D color Doppler of fetal brain circulation

\section{VCI (Volume Contrast Imaging)}

VCI is a unique new method to generate contrast-enriched real time 2D images by utilizing 4D volume scan technology. "Thick Slice" imaging uses volume ultrasound capabilities that renders from slices in a volume enhanced contrast and speckle suppression, a better assessment of size, margins and internal structures of lesions. The result is an image with no speckle pattern and a highly improved tissue contrast (Fig. 4). It can be used on two different planes: A-plane (VCI-A) and C-plane (VCI-C).

\section{SRI (SPECKLE REDUCTION IMAGING, GE) - DYNAMIC MR (MEDISON)}

Speckle Reduction Imaging is a technological innovation improving both contrast resolution and border detection. At the same time the unwanted ultrasound characteristic patterns (SPECKLE) are greatly reduced. This new enhanced algorithm boosts image quality and clinical confidence, which allows the reduction of noise and speckle in homogeneous regions.

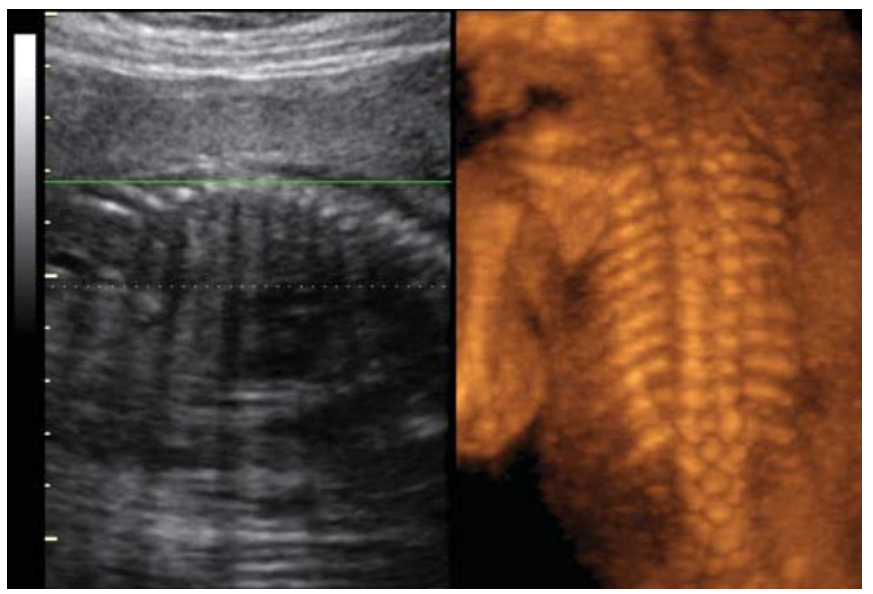

Fig. 4: $\mathrm{VCl}-\mathrm{C}$ technique for the examination of fetal spine and ribs in maximum mode

Tissues of various organs have their own specific acoustic properties, so different tissues and bone structures need to be treated differently. This technique allows for an enhancement of object boundaries and small physiological features.

\section{Magic Cut}

It clears the overlapping structures to get a high quality surface image with the electronic scalpel, which removes the disturbing echoes.

\section{Volume Calculation}

For volume measurement, software tools such as VOCAL are available. There are several methods for measuring the volume of structures of varying shapes. The measured contour can be defined automatically or manually in different planes and images. After the rotation the volume can be easily calculated. 


\section{THE ROLE OF 3D/4D ULTRASOUND IN DETECTION OF FETAL MALFORMATIONS}

\section{Central Nervous System}

Three-dimensional ultrasonography can acquire the volume of the fetal head and brain in any position. The neurosonographic technique was described some years ago by Monteagudo and Timor-Tritsh, ${ }^{13}$ and then ISUOG issued guidelines for performing the "basic examination" and the "fetal neurosonogram". 7 The multiplanar imaging facilitates imaging of the transventricular, the transcerebellar and transthalamic planes revealing the normal or abnormal anatomy of the fetal brain. For a detailed neurosonogram, which is designed to diagnose the pathologic brain structures and malformations it is essential to assess also the coronal, the sagittal and parasagittal planes. ${ }^{7}$

The most important advantages of 3D ultrasound in detection of anomalies of fetal head:

- Malformations of midline cerebral structures and posterior fossa abnormalities,

- Malformations of cortical development,

- Identification of fetal face anomalies,

- Sonographic markers of chromosomal abnormalities,

- 4D functional examination of fetal face and behavior.

Prenatal sonographic diagnosis of craniosynostosis is based on the identification the premature closure of the sutures and the associated deformation of the skull. The metopic suture was examined by Faro et $\mathrm{al}^{3}$ in fetuses with Apert syndrome. Their findings demonstrate that the characteristic features of frontal bossing and midfacial hypoplasia are associated with closure of the coronal sutura and a wide gap between the frontal bones.

Evaluation of the structures of posterior fossa, the cisterna magna and cerebellar vermis is particularly useful in the detection of various abnormalities such as neural tube defects, DandyWalker complex and Joubert syndrome. ${ }^{21}$

Three-dimensional ultrasonography can acquire an accurate volume in any position and then multiplanar technique can be used to obtain an appropriate view for the examination of midline structures, especially the corpus callosum. ${ }^{17}$ Pilu et al investigated the effectiveness of a simplified approach for evaluation of midline structures of the fetal brain using 3D ultrasound, and concluded that the 3D allows an accurate diagnosis of normal cerebral anatomy and anomalies of corpus callosum and cerebellar vermis. ${ }^{14,16}$

A group from the Netherlands explored three-dimensional sonography of prenatal skull base development. The skull base has an important role in supporting and inducing the formation of craniofacial structures, so the measurement and evaluation of the anterior and posterior skull base may help to understand the background of some anomalies. ${ }^{19}$

In some cases the ultrasound can be superior to digital examination: visualization of the fetal fontanels and skull sutures using 3D translabial ultrasound during the second stage of labor is the latest development by Fuchs et al. ${ }^{6,7}$ Identification of the position of the fetal head and the exact degree of internal rotation are essential for a safe vaginal instrumental delivery.

Trisomy 21 has well-known characteristics on the fetal face: signs not only include the missing nasal bone and increased nuchal translucency in the first trimester, but a flat face, which can now be quantified by measurement of the frontomaxillary facial (FMF) angle. ${ }^{2,18}$ Most information that has been reported on nasal bone and NT and its association with Down syndrome is well-documented. Recently it has been demonstrated that a unilateral absence of nasal bone is also associated with Down syndrome. The use of maximum mode can be helpful in detection of bony structures (Figs 5A to $\mathrm{C}$ ), which allows improved counseling and aneuploidy risk assessment of pregnancy. ${ }^{1}$

Paladini et al recently described the methodology to obtain correct visualization of fetal anterior fontanelle. The fact that enlarged fontanelle dimensions may be associated with certain fetal abnormalities may be employed in the differential diagnosis of some syndromic conditions or aneuploides. ${ }^{15}$

The surface of the developing fetal brain undergoes significant morphological changes throughout gestation. It is important to know this physiologic process in order to understand and detect the malformations of cortical development (MCD). ${ }^{5,13}$ Cerebral cortical development is an extremely complex process, comprising of three major, and overlapping steps: cell proliferation, neuronal migration and cortical organization. The new ultrasound technique made it possible to detect minor alterations of myelinization of the fetal brain and disturbed development of sulci and gyri. Lissencephaly can be diagnosed prenatally like other rare developmental disorders from impaired neuronal migration. ${ }^{5,10}$

With the introduction of 3D/4D ultrasonography there is no clear evidence supporting the assumption that an MRI is superior to state of the art ultrasound in the diagnosis of most common brain anomalies. ${ }^{9}$ An MRI may provide verification in fetuses with isolated ultrasonographic findings and give additional information in rare cases with migration disorders. ${ }^{11}$

\section{Face}

In the second and third trimester, the multiplanar display mode is helpful in identifying the abnormal fetal profile and surface rendering adds more precise information and gives a clear demonstration of all defects on the fetal surface, such as a cleft lip, and ear and eye abnormalities. ${ }^{12}$ 

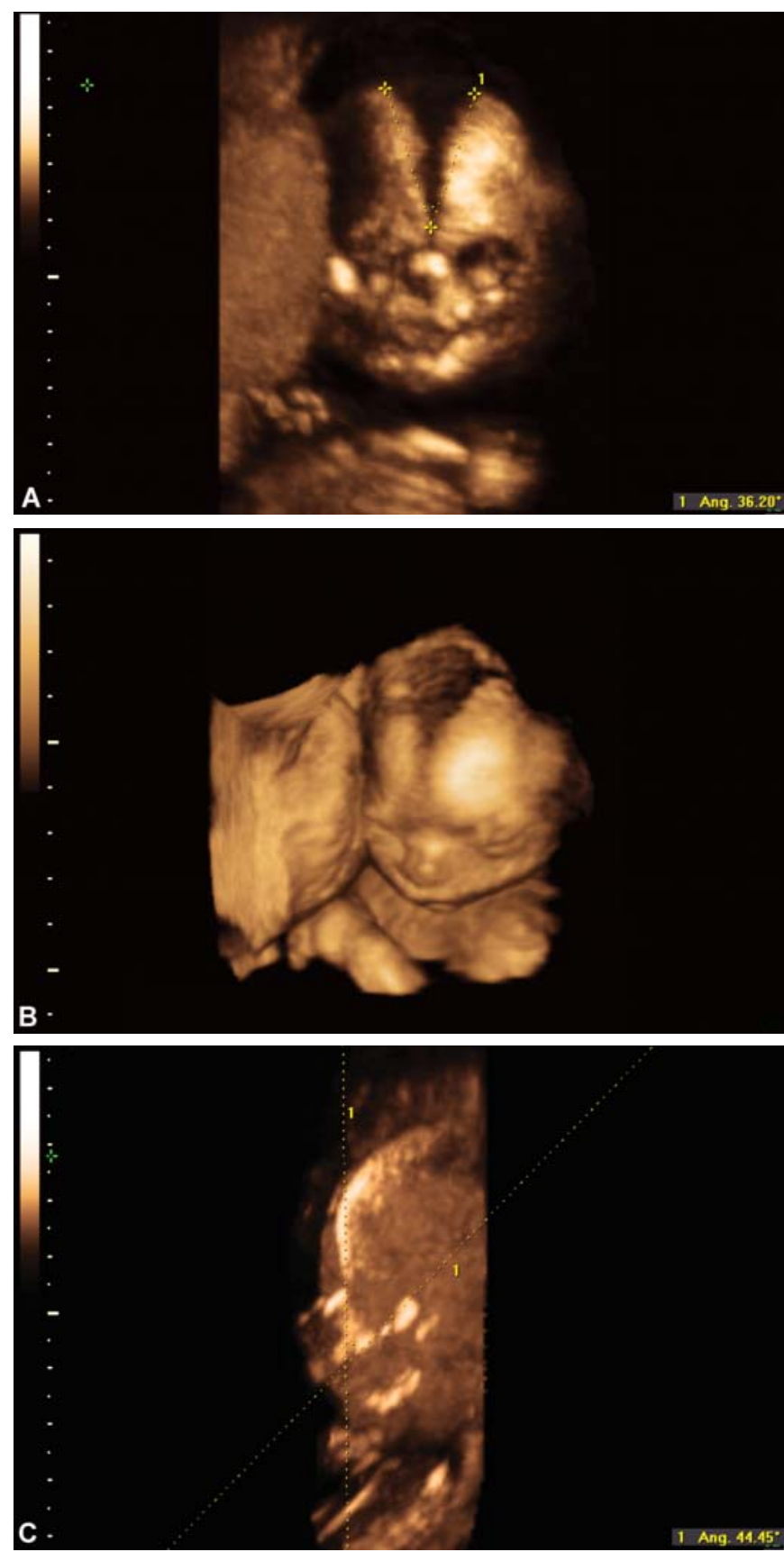

Figs 5A to C: Analysis of frontomaxillar angle, and assessment of metopic suture using multiplanar mode

The use of the 3D technique in assessing the normal and abnormal fetal face is well-studied. We cannot forget that the effect of bonding between parents and the fetus may have some positive impact, so the surface mode on face is most frequently used to show the "baby" to the parents. The multiplanar mode is used as a diagnostic tool. ${ }^{20}$ Visualization of different structures such as midline defects, palate, eye abnormalities, nasal bone, and angulation of the jaw are possible in the multiplanar mode after the volume has been rotated into a symmetric orientation.

Imaging of the fetal palate is subject to several technical difficulties, such as shadowing effects of the superior alveolar ridge, and the fetal prone position. ${ }^{4}$ However, 3D ultrasound has been able to detect the hard palate, but the evaluation of the soft palate still remains a difficult task. French investigators suggest a $30^{\circ}$-inclined axial 3D ultrasound view to get the best result in assessing the integrity of the fetal soft palate. ${ }^{4}$

\section{Fetal Neurobehavior}

Kurjak's preliminary work using 3D/4D ultrasound as an assessment of fetal neurological status may help in detecting fetal brain and neurodevelopmental alterations due to in utero brain impairment. It gives a new aspect to the traditional concept of the fetal biophysical profile score. Isolated head anteflexion, eye blinking, facial alteration (grimace or tongue expulsion), and mouth opening (yawning or mouthing) are used for the new scoring system ${ }^{8}$ (Figs 6A and B).

\section{Heart}

Spatiotemporal image correlation (STIC) is a new approach for fetal heart diagnosis, using a 4D real time dataset presenting one heart cycle in motion. STIC is a technique that allows quick capture of a full fetal heart cycle beating in real-time and saving the volume for later analysis.

\section{EXTREMITIES}

The different rendering modalities give a clear display of extremities, enabling an assessment of skeletal development and related abnormalities, examination of curved skeleton parts such as the spine, ribs and skull, and it emphasizes the visualization of the complex anatomy of bony structures. Especially in this field, using the 3D/4D technique allows to diagnose and differentiate rare musculoskeletal dysplasias, focal limb and spine anomalies, clubfoot deformity (Fig. 7), and small neural tube defects. Long bones, clavicles, cranial structures and the spine can be evaluated using maximum mode or by increasing the threshold of the surface-rendering mode. Ribs can be easily counted, and the level of spinal defect can be potentially predicted. Rotating the volume is a useful tool for determining the type and position of the defect. ${ }^{20}$

\section{CONCLUSION}

Sometimes the fetal position, a small amount of amniotic fluid, creation of a false-positive result by shadowing will cause some 

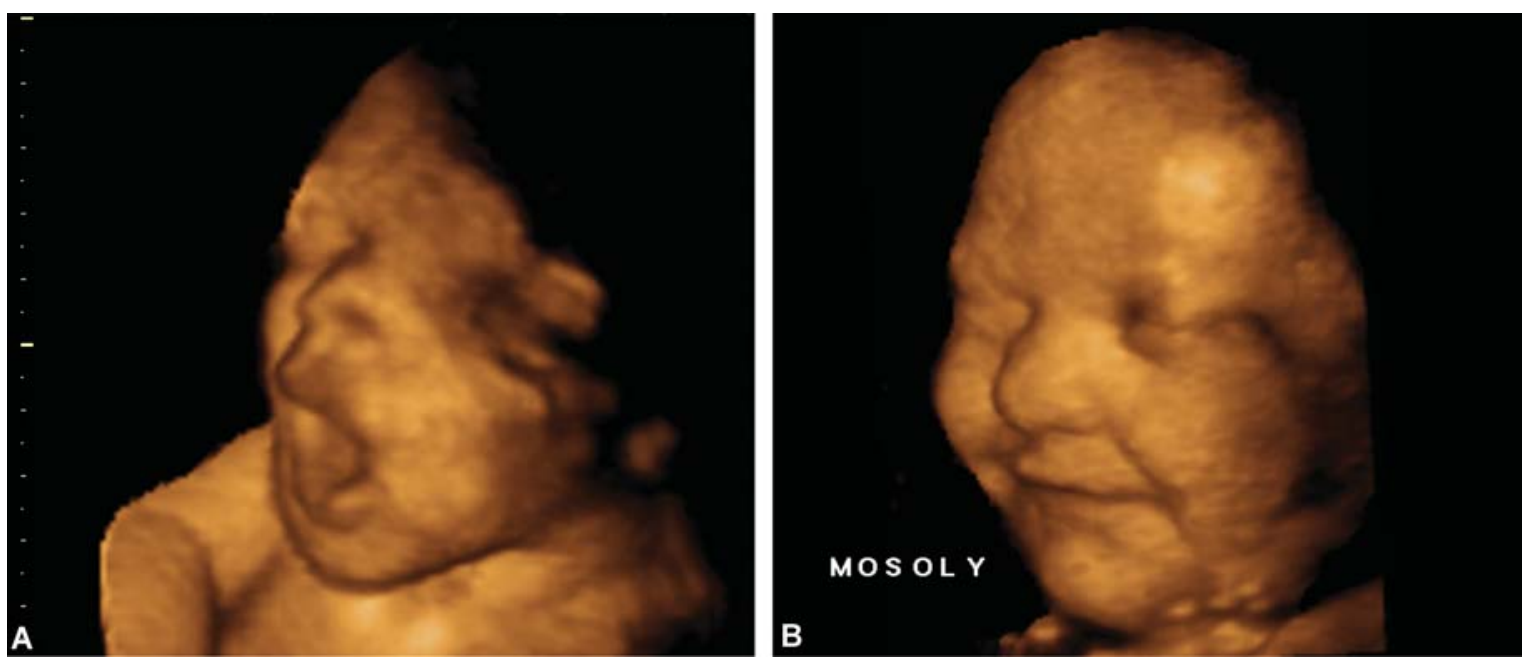

Figs 6A and B: Fetal yawning and smiling as a marker of fetal neurobehavior or biophysical assessment (Courtesy: Dr Tankó)

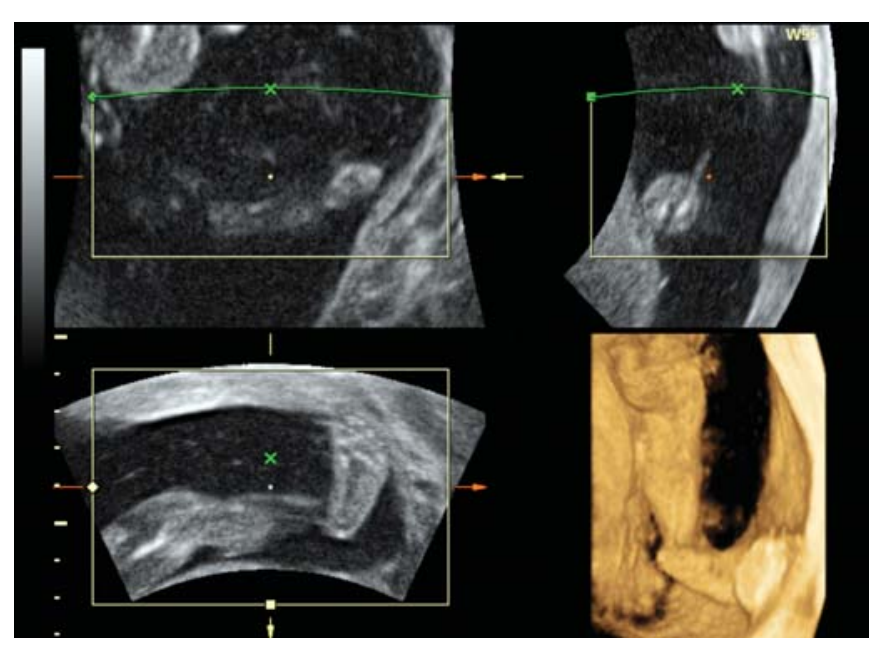

Fig. 7: Multiplanar mode of fetal clubfoot diagnosed in the second trimester of pregnancy

limitations, and challenge the experience and skill of the operator. Though the traditional 2D ultrasound with high resolution provides a great diagnostic tool in detection of fetal malformations, there is no doubt that the $3 \mathrm{D} / 4 \mathrm{D}$ technique offers a new power in prenatal diagnosis. It was demonstrated that the 3D ultrasound could even detect subtle defects that were not found with a conventional 2D. Merz and Welter found that the 3D ultrasound provided conclusive detection of fetal defects in $4.2 \%$ of cases (42 of 1012), whereas the 2D technique failed to provide this information. ${ }^{12}$ The multiplanar imaging gives a view of all scan planes and enables the visualization of different fetal structures. All planes of view are reproducible and the examination time is reduced, which decreases the patient waiting times and makes the procedure faster. It has eliminated the limitations of 2D scanning and the normal and abnormal fetal anatomy has become more distinguishable than previously. Three-dimensional ultrasound can assist in the diagnosis of different, rare malformations because it offers a potential benefit of understanding spatial relationships of normal and abnormal fetal anatomy. The images are more readily recognized by parents and anomalies are easier to explain. Volume data can be stored and reviewed later.

\section{REFERENCES}

1. Benoit B, Chaoui R. Three-dimensional ultrasound with maximal mode rendering: A novel technique for the diagnosis of bilateral or unilateral abscence or hypoplasia of nasal bones in secondtrimester screening for Down syndrome. Ultrasound Obstet Gynecol 2005;25:19-24.

2. Borenstein M, Persico N, Kagan KO, et al. Frontomaxillary facial angle in screening for trisomy 21 at $11^{+0}$ to $13^{+6}$ weeks. Ultrasound Obstet Gynecol 2008;32:5-11.

3. Faro C, Chaoui R, Wefrzyn P, et al. Metopic suture in fetuses with Apert syndrome at 22-27 weeks of gestation. Ultrasound Obstet Gynecol 2006;27:28-33.

4. Faure JM, Baumler M, Boulot P, et al. Prenatal assessment of the normal fetal soft palate by three-dimensional ultrasound examination: Is there an objective technique? Ultrasound obstet Gynecol 2008;31:652-56.

5. Fong KW, Ghai S, Toi A, et al. Prenatal ultrasound findings of lissencephaly associated with Miller-Dieker syndrome and comparison with pre- and postnatal magnetic resonance imaging. Ultrasound Obstet Gynecol 2004;24:716-23.

6. Fuchs I, Tutschek B, Heinrich W. Visualization of the fetal fontanels and skull sutures by three-dimensional translabial ultrasound during the second stage of labor. Ultrasound Obstet Gynecol 2008;31:484-86. 
7. ISUOG Guidelines. Sonographic examination of the fetal central nervous system: Guidelines for performing the "basic examination" and the "fetal neurosonogram”. Ultrasound Obstet Gynecol 2007;29:109-16.

8. Kurjak A, Miskovic B, Stanojevic M, et al. New Scoring Sytem for fetal neurobehavior assessed by three- and four-dimensional sonography. J Perinat Med 2008;36:73-81.

9. Malinger G, Ben-Sira L, Lev D, et al. Fetal brain imaging: A comparison between magnetic resonance imaging and dedicated neurosonography. Ultrasound Obstet Gynecol 2004;23:333-40.

10. Malinger G, Kidron D, Schreiber L, et al. Prenatal diagnosis of malformations of cortical development by dedicated neurosonography. Ultrasound Obstet Gynecol 2007;29:178-91.

11. Malinger $G$, Lev D, Lerman-Sagie T. Is fetal magnetic resonance imaging superior to neurosonography for detection of brain anomalies? Ultrasound Obstet Gynecol 2002;20:317-21.

12. Merz E. Current 3D/4D ultrasound technology in prenatal diagnosis. Eur Clinics Obstet Gynecol 2006; DOI 10.1007/ s11296-005-0005-6.

13. Monteagudo A, Timor-Tritsch IE: Development of fetal gyri, sulci and fissures: A transvaginal sonographic study. Ultrasound Obstet Gynecol 1997;9:222-28.

14. Monteagudo A, Timor-Tritsch IE, Mayberry P. Threedimensional transvaginal neurosonography of the fetal brain: "Navigating" in the volume scan. Ultrasound Obstet Gynecol 2000;16:307-13.
15. Paladini D, Vassallo M, Sglavo G, et al. Normal and abnormal development of the fetal anterior fontanelle: A three-dimensional ultrasound study. Ultrasound Obstet Gynecol 2008;DOI: 10.1002/uog.5368.

16. Pilu G, Segata M, Ghi T, et al. Diagnosis of midline anomalies of the fetal brain with the three-dimensional median view. Ultrasound Obstet Gynecol 2006;27:522-29.

17. Plasencia W, Dagklis T, Borenstein $\mathrm{M}$ et al. Assessment of the corpus callosum at 20-24 weeks' gestation by three-dimensional ultrasound examination. Ultrasound Obstet Gynecol 2007;30:169-72.

18. Plasencia W, Dagklis T, Pachoumi C, et al. Frontomaxillary facial angle in screening for trisomy 21 at $11^{+0}$ to $13^{+6}$ weeks: effect of plane of aquisition. Ultrasound Obstetrics Gynecol 2007;29:660-65.

19. Roelfsema NM, Drijseels EWM, Hop WCJ, Wladimiroff JW: Three-dimensional sonography of prenatal skull base development. Ultrasound Obstet Gynecol 2007;DOI:10.1002/ uog.3946.

20. Tache V, Tarsa M, Romine L, Pretorius DH: Three-dimensional obstetric ultrasound. Semin Ultrasound CT MRI 2008;29:14755.

21. Vinals F, Munoz M, Naveas R, et al. The fetal cerebellar vermis: Anatomy and biometric assessment using volume contrast imaging in the C-plane (VCI-C). Ultrasound Obstet Gynecol 2005;26:622-27. 